International Journal of Pure and Applied Mathematics

Volume 104 No. 1 2015, 1-18

ISSN: 1311-8080 (printed version); ISSN: 1314-3395 (on-line version)

url: http://www.ijpam.eu

doi: http://dx.doi.org/10.12732/ijpam.v104i1.1

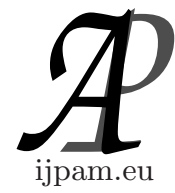

\title{
HOPF BIFURCATION ANALYSIS OF ZHOU SYSTEM
}

\author{
Tee Loong Soon ${ }^{1}$, Zabidin Salleh ${ }^{2} \S$ \\ ${ }^{1}$ HELP Matriculation Centre \\ HELP University \\ Kompleks Pejabat Damansara (KPD), Jalan Dungun, \\ 50490, Kuala Lumpur, MALAYSIA \\ ${ }^{2}$ School of Informatics and Applied Mathematics \\ Universiti Malaysia Terengganu \\ 21030, Kuala Terengganu, Terengganu, MALAYSIA
}

\begin{abstract}
In this paper, we conducted an extension research from [20] which was mainly on studying the dynamical behaviour such as Lyapunov exponents, bifurcation diagram, etc. of a chaotic attractor namely Zhou System. In order to understand the Zhou system more depth, we presented the analysis of the system by using Complementary-Cluster Energy-Barrier Criterion (CCEBC) and Routh-Hurwitz Criterion. Besides that, we have demonstrated the occurrence of Hopf bifurcation. The results showed that the Zhou system undergoes a supercritical Hopf bifurcation under a special condition by means of the first Lyapunov coefficient.
\end{abstract}

AMS Subject Classification: 34C23, 34C28, 37M20

Key Words: dynamical system, chaos, Zhou system, Hopf bifurcation

\section{Introduction}

Hopf bifurcation has been studied intensively in the field of dynamical system by mathematicians lately. One of the latest researches on Hopf bifurcation was presented by Yan [18] in a Lorenz-type chaotic system. In developing Hopf bifurcation research, Li and Jin [9] have demonstrated Hopf bifurcation

Received: March 19, 2015

(C) 2015 Academic Publications, Ltd.

$\S_{\text {Correspondence author }}$ url: www.acadpubl.eu 
analysis and numerical analysis on a four-dimensional hyperchaotic system. They showed a detailed set of conditions which guarantees the existence of the Hopf bifurcation. The standard normal form theory was applied to determine the direction and type of the Hopf bifurcation, and the approximate expressions of bifurcating periodic solutions and their periods. Furthermore, the theoretical results are justified by numerical simulations.

$\mathrm{Wu}$ et al. [17], on the other hand, have presented another new fourdimensional autonomous system, which has a complex hyperchaotic dynamics. They utilized the Kaplan-Yorke dimension, Poincaré sections and the frequency spectra to demonstrate the complexity of such hyperchaotic attractor. They analysed the local bifurcation in the system, so that a Hopf bifurcation is proved to occur when the appropriate bifurcation parameter passes the critical value.

More researches on Hopf bifurcation were done such as the Hopf bifurcation on Rossler chaotic system with delayed feedback [1], Hopf bifurcation of a unified chaotic system - the generalised Lorenz canonical form (GLCF) [10], and Hopf bifurcation of the Chen system [11]. Besides that, one of the latest research in $[15,16]$ about Hopf bifurcation on a modified Lorenz system was carried recently by Tee and Salleh, which resulted an interesting results.

There are a lot of studies on Hopf bifurcation in predator-prey models. Many examples and different models were presented in the recent journals such as the delayed predator-prey system [6], the delayed predator-prey system with diffusion effects [5], the predator-prey system with a discrete delay and a distributed delay [19], and many more [2, 14].

In order to understand the Zhou system in more depth, we presented the dynamical analysis and Hopf bifurcation of the system in more details. In his paper [20], he has studied some basic dynamical properties, such as Lyapunov exponents, Poincaré mapping, fractal dimension, bifurcation diagram, continuous spectrum and chaotic dynamical behaviours. While Roslan et al. [12] has discussed the matter in solving Zhou system using Euler's method. It is one of the simplest approaches to obtain the numerical solution of a differential equation. Recently Roslan et al. [13] has solved Zhou system using Runge-Kutta method. This method is more efficient as compared to the Euler's method to analyse the system.

In Zhou's paper, the presented Zhou system is as follow:

$$
\begin{aligned}
& \dot{x}=a(y-x) ; \\
& \dot{y}=-x z+b x ; \\
& \dot{z}=x y+c z ;
\end{aligned}
$$

where $x, y$, and $z$ are variables and $a, b$, and $c$ are real constant parameters. 
In this paper, we investigated dynamical analysis of the system (1) with the Routh-Hurwitz Criterion and Complementary-Cluster Energy-Barrier Criterion (CCEBC) [11] as well. Furthermore, we demonstrated the Hopf bifurcation of the system (1) by using the first Lyapunov coefficient [8]. Lastly, some of the phase portraits of the Zhou system are plotted when Hopf bifurcation occurs at critical values.

\section{Some Basic Dynamical Properties of the Zhou System}

In this section, we discussed some further properties of the Zhou system. Let $x=y=z=0$ in the system (1), and then we obtained the following equilibrium points:

$$
\left\{\begin{array}{c}
P_{1}=(0,0,0) \\
P_{2}=(\sqrt{(-b c)}, \sqrt{(-b c)}, b) \\
P_{3}=(-\sqrt{(-b c)},-\sqrt{(-b c)}, b) .
\end{array}\right.
$$

Next, we linearized the system (1) by letting:

$$
\left\{\begin{array} { l } 
{ X = x - x _ { 0 } ; } \\
{ Y = y - y _ { 0 } ; } \\
{ Z = z - z _ { 0 } }
\end{array} \Rightarrow \left\{\begin{array}{l}
x=X+x_{0} \\
y=Y+y_{0} \\
z=Z+z_{0}
\end{array}\right.\right.
$$

where $\left(\mathrm{x}_{0}, \mathrm{y}_{0}, \mathrm{z}_{0}\right)$ is any nontrivial equilibrium point, and the system (1) becomes

$$
\left\{\begin{array}{l}
\dot{X}=a\left(Y-X+y_{0}-x_{0}\right) \\
\dot{Y}=-\left(X Z+X z_{0}-Z x_{0}-z_{0} x_{0}\right)+b X-b x_{0} \\
\dot{Z}=X Y+X y_{0}+Y x_{0}+x_{0} y_{0}+c Z+c z_{0}
\end{array}\right.
$$

The general Jacobian matrix from the system (1) is given by

$$
D F=\left[\begin{array}{ccc}
-a & a & 0 \\
b-z & 0 & -x \\
y & x & c
\end{array}\right] .
$$

When the equilibrium point is $P_{1}(0,0,0)$, the characteristic equation of $(5)$ is

$$
(\lambda-c)\left(\lambda^{2}+a \lambda-a b\right)=0 .
$$

Thus, by solving the equation (6), we obtain the three eigenvalues that are

$$
\lambda_{1}=c,
$$




$$
\begin{aligned}
& \lambda_{2}=\frac{-a-\sqrt{\left(a^{2}+4 a b\right)}}{2}, \\
& \lambda_{3}=\frac{-a+\sqrt{\left(a^{2}+4 a b\right)}}{2} .
\end{aligned}
$$

Observe that if $a>0$, then $\lambda_{2}$ is always negative.

As for the equilibrium points $P_{2}=(\sqrt{(-b c)}, \sqrt{(-b c)}, b)$ or $P_{3}=(-\sqrt{(-b c)}$, $-\sqrt{(-b c)}, b)$, the characteristic equation is

$$
\lambda^{3}+(a-c) \lambda^{2}-(b c+a c) \lambda-2 a b c=0 .
$$

The equation (10) always has one real negative root and a pair of complex conjugate roots when $a>0, b>0$ and $c<0$. It is difficult to determine the behaviour of the equilibrium point since the equation (10) has a degree three and difficult to solve. However, by using Routh-Hurwitz Criterions, we can lead to the conclusion either the real parts of the roots $\lambda$ are negative or not. Since the two equilibrium points, $P_{2}=(\sqrt{(-b c)}, \sqrt{(-b c)}, b)$ and $P_{3}=(-\sqrt{(-b c)},-\sqrt{(-b c)}, b)$ have the same stability due to the symmetrical property, it is sufficient to analyse the point $P_{2}=(\sqrt{(-b c)}, \sqrt{(-b c)}, b)$ only. Let

$$
\begin{aligned}
& A=(a-c), \\
& B=-(b c+a c), \\
& C=-2 a b c .
\end{aligned}
$$

Lemma 1. [3] (Routh-Hurwitz Criterion) Let

$$
F(\lambda)=x_{n} \lambda^{n}+x_{n-1} \lambda^{n-1}+x_{n-2} \lambda^{n-2}+\cdots+x_{1} \lambda+x_{0}
$$

where $x_{i}, i=0,1, \ldots, n$ are the coefficients and $\lambda$ are the eigenvalues. Define the Hurwitz matrices using the coefficients $x_{i}$ of the equation:

$$
\begin{gathered}
H_{1}=\left[x_{n-1}\right], \quad H_{2}=\left[\begin{array}{ccc}
x_{n-1} & x_{n} \\
x_{n-3} & x_{n-2}
\end{array}\right], \\
H_{3}=\left[\begin{array}{cccc}
x_{n-1} & x_{n} & 0 \\
x_{n-3} & x_{n-2} & x_{n-1} \\
x_{n-5} & x_{n-4} & x_{n-3}
\end{array}\right], \\
H_{n}=\left[\begin{array}{cccccc}
x_{n-1} & x_{n} & 0 & 0 & \ldots & 0 \\
x_{n-3} & x_{n-2} & x_{n-1} & x_{n} & \ldots & 0 \\
x_{n-5} & x_{n-4} & x_{n-3} & x_{n-2} & \ldots & 0 \\
\vdots & \vdots & \vdots & \vdots & \ldots & \vdots \\
0 & 0 & 0 & 0 & \ldots & x_{0}
\end{array}\right] .
\end{gathered}
$$


All of the roots of the equation (12) are negatives or have negative real parts if and only if the determinants of all the Hurwitz matrices are positive:

$$
\operatorname{det}\left(H_{j}\right)>0, j=1,2, \ldots, n .
$$

From the definition above, we applied Routh-Hurwitz Criterion onto the equation (10) to get the following matrices:

$$
\begin{gathered}
H_{1}=[a-c], \quad H_{2}=\left[\begin{array}{cc}
a-c & 1 \\
-2 a b c & -(b c+a c)
\end{array}\right], \\
H_{3}=\left[\begin{array}{ccc}
a-c & 1 & 0 \\
-2 a b c & -(b c+a c) & a-c \\
0 & 0 & -2 a b c
\end{array}\right] .
\end{gathered}
$$

By using the Routh-Hurwitz Criterion, the equation (10) has all negative real parts of $\lambda$ if and only if:

$$
\left.\begin{array}{rl}
(a-c) & >0 \\
-2 a b c & >0 \\
-(a-c)(b c+a c)+2 a b c & >0
\end{array}\right\} .
$$

Theorem 2. The equilibrium points $P_{2}=(\sqrt{(-b c)}, \sqrt{(-b c)}, b)$ and $P_{3}=$ $(-\sqrt{(-b c)},-\sqrt{(-b c)}, b)$ are asymptotically stable if and only if (13) holds.

Furthermore, in the equation (10), let

$$
f(\lambda)=\lambda^{3}+(a-c) \lambda^{2}-(b c+a c) \lambda-2 a b c=0 .
$$

Notice that all the coefficients of the equations (14) are all positive if $c$ is a negative value. So, we can conclude that $f(\lambda)>0$ for all $\lambda \geq 0$ and $a>0, b>0$ and $c<0$. One of the eigenvalues is always a negative real number because $f(\lambda)>0$ at $\lambda=0$ and $f(\lambda) \rightarrow-\infty$ as $\lambda \rightarrow-\infty$. Hence, there is instability in the Zhou system if there are two complex conjugate zeros of $f$. Assuming $\lambda_{1}=i \omega$ and $\lambda_{2}=-i \omega$ for some real $\omega$, the sum of three zeros of the function $f$ can be obtained as

$$
\lambda_{1}+\lambda_{2}+\lambda_{3}=-(a-c)
$$

and we have that $\lambda_{3}=-(a-c)$, which is on the margin of stability. On this margin, we have the following equation

$$
0=f(-(a-c))=c\left[a^{2}-(c+b) a-b c\right]
$$

that is,

$$
a_{1,2}=\frac{(c+b) \pm \sqrt{c^{2}+6 b c+b^{2}}}{2}
$$




\section{Dynamical Analysis Using Complementary-Cluster Energy-Barrier Criterion (CCEBC)}

We determined the dynamical behaviours of Zhou system in the mean of complementary-cluster energy-barrier criterion or also known as CCEBC [11]. From Zhou system, we let the first two differential equations be:

$$
\left\{\begin{array}{l}
\dot{x}=a(y-x) \\
\dot{y}=-x z+b x
\end{array}\right.
$$

where $z=z(t)$ that is a known function of time variable, $t$. As for $a$ and $b$ are the real constant parameters with the case of $t=t_{o}$. So, the system (18) is a two-dimensional linear system with constant coefficients which the system itself is simple and global. We obtained the Jacobian matrix as follow:

$$
J=\left[\begin{array}{cc}
-a & a \\
b-z & 0
\end{array}\right]
$$

The system (18) gives the following characteristic equation at the origin $(0,0)$ :

$$
\lambda^{2}+a \lambda+a(b-z)=0 .
$$

Thus, we have analysed the above equation under certain circumstances:

1. When $a>0, b>0$ and $z>b$, the system (18) has two real eigenvalues such that $\lambda_{1}<0<\lambda_{2}$. This implies that the fixed point $(0,0)$ is a saddle point in the two-dimensional plane. (Figure 1A)

2. When $a>0, b>0$, and $b-a / 4<z<b$, the system (18) has two negative real eigenvalues such that $\lambda_{1}<0, \lambda_{2}<0$. This implies that the fixed point $(0,0)$ is a stable node point in the two-dimensional plane. (Figure 1B)

3. When $a>0, b>0$, and $z>b-a / 4$, the system (18) has two complex eigenvalues such that $\lambda_{1,2}=\alpha+\beta i$ with negative real part. This implies that the fixed point $(0,0)$ is a stable focus in the two-dimensional plane. (Figure 1C)

4. When $a>0, b>0$, and $z=b-a / 4$, the system (18) has two repeated real eigenvalues such that $\lambda_{1,2}=\alpha$ (negative). This implies that the fixed point $(0,0)$ is a degenerate stable node in the two-dimensional plane. (Figure 1D) 
5. When $a<0, b>0$, and, $b-a / 4<z<b$ the system (18) has two positive real eigenvalues such that $\lambda_{1}>0, \lambda_{2}>0$. This implies that the fixed point $(0,0)$ is an unstable node point in the two-dimensional plane. (Figure 1E)

6. When $a<0, b>0$, and, $z<b-a / 4$ the system (18) has two complex eigenvalues such that $\lambda_{1,2}=\alpha+\beta i$ with positive real part. This implies that the fixed point $(0,0)$ is an unstable focus in the two-dimensional plane. (Figure $1 \mathrm{~F}$ )

7. When $a<0, b>0$, and $z=b-a / 4$, the system (18) has two repeated real eigenvalues such that $\lambda_{1,2}=\alpha$ (positive). This implies that the fixed point $(0,0)$ is a degenerate unstable node in the two-dimensional plane. (Figure $1 \mathrm{G}$ )

8. When $a<0$ and $z=b$, the system (18) has the first eigenvalue equal to zero and the second eigenvalue is positive such that $\lambda_{1}=0, \lambda_{2}>0$. (Figure 1H)

9. When $a=0$ and $z=b$, the system (18) has the first eigenvalue equal to zero and the second eigenvalue is equal to zero also such that $\lambda_{1,2}=0$.

10. When $a>0$ and $z=b$, the system (18) has the first eigenvalue equal to zero and the second eigenvalue is negative such that $\lambda_{1}=0, \lambda_{2}<0$. (Figure 1I)

Figure 2 is the chaotic time series for the function $z(t)$ with two straight lines $z(t)=b$ (solid) and $z(t)=b-a / 4$ (long dash). It is obvious that as time the function $z(t)$ passes through the straight line alternatively as the time, $t$ goes to infinity. The $z(t)$ has divided into three partitioned disjointed domains: $(-\infty, b-a / 4),(b-a / 4, b)$, and $(b, \infty)$. Hence, the system (18) has different dynamical behaviours in the stated different domains. The dynamical behaviour changes in the system (18) and passes through these domains repeatedly, leading to a complicated dynamical behaviour when the time, $t$ goes to infinity.

\section{Hopf Bifurcation Analysis}

For Hopf bifurcation analysis, we used the method of $[4,8,16]$. We preferred to discuss the matter using scalar product [7]. However, only the equilibrium point $P_{2}=(\sqrt{(-b c)}, \sqrt{(-b c)}, b)$ will be studied and discussed here due to the symmetrical properties of $P_{2}=(\sqrt{(-b c)}, \sqrt{(-b c)}, b)$ and $P_{3}=$ $(-\sqrt{(-b c)},-\sqrt{(-b c)}, b)$. 


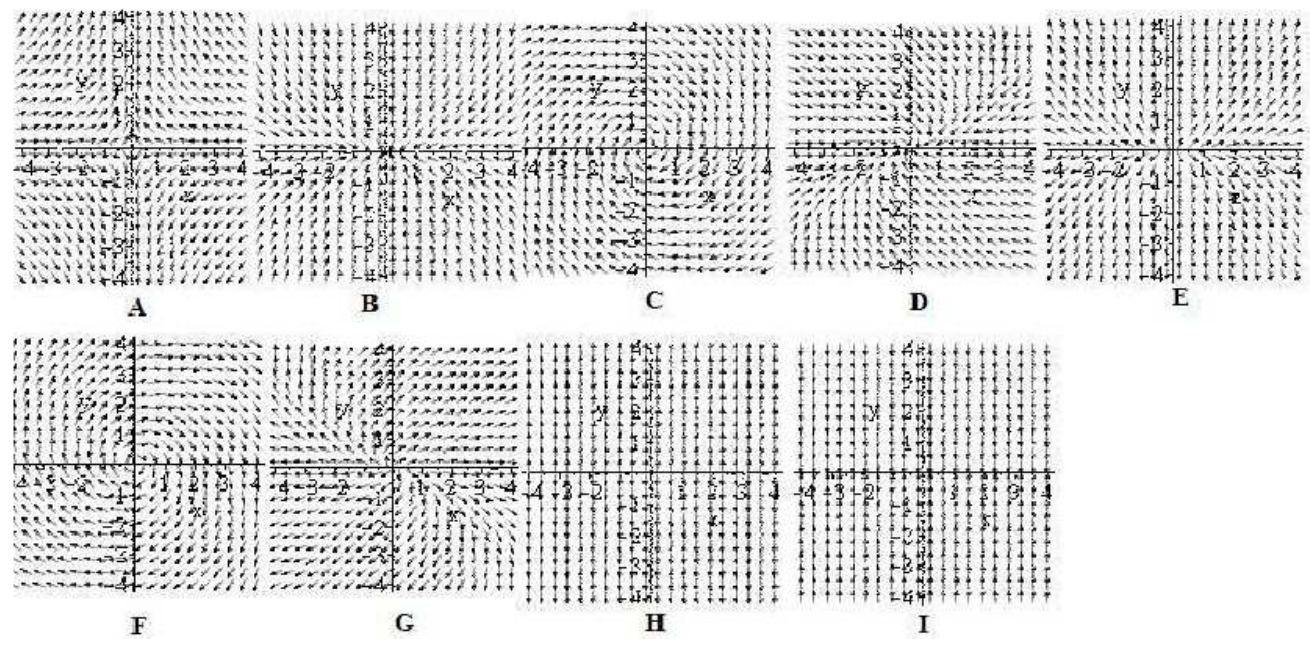

Figure 1: The possible phase portraits for system (18): A) saddle point, B) stable node point, C) stable focus, D) degenerate stable node, E) unstable node point $\mathrm{F}$ ) unstable focus $\mathrm{G}$ ) degenerate unstable node $\mathrm{H}$ ) $\lambda_{2}>0$ and I) $\lambda_{2}<0$.

The stability of the steady state of the equilibrium point $P_{2}=(\sqrt{(-b c)}$, $\sqrt{(-b c)}, b)$ is analyzed by linearising the system (1). Hence, it undergoes linear transformation and yields the following characteristic equation same with equation (14):

$$
\lambda^{3}+(a-c) \lambda^{2}-(b c+a c) \lambda-2 a b c=0 .
$$

From equations (13), Hopf bifurcation will appear when $P_{2}=(\sqrt{(-b c)}, \sqrt{(-b c)}$, $b$ ) loses its stability. It will happen if $-(a-c)(b c+a c)+2 a b c=0$, that is,

$$
b c^{2}+a c^{2}+a b c-c a^{2}=0
$$

which yields as follow,

$$
b=\frac{a^{2}-a c}{a+c}=b_{0} .
$$

Next, by assuming that the equation (21) has a pair of imaginary roots, thus by substituting $\lambda=\omega i, \lambda^{2}=-\omega^{2}, \lambda^{3}=-\omega^{3} i$ into equation (21) we have:

$$
-\omega^{3} i-(a-c) \omega^{2}-(b c+a c) \omega i-2 a b c=0 .
$$




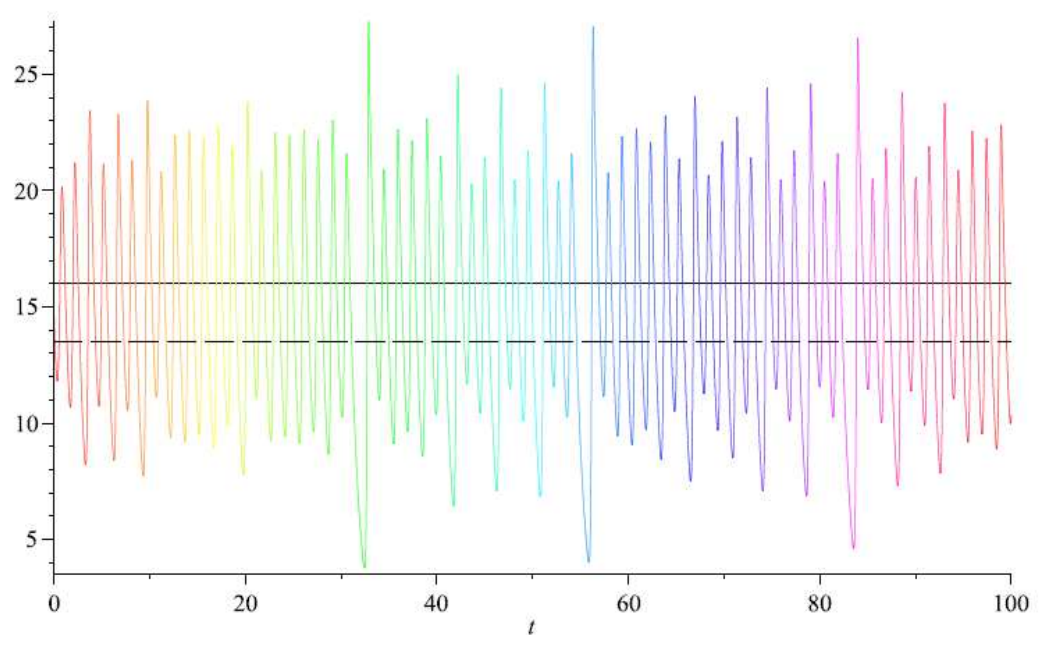

Figure 2: The chaotic time series of $z(t)$ when $a=10, b=16$ and $c=-1$ with two straight lines $z=16$ (solid) and $z=13.5$ (long dash).

Then, equation (24) becomes:

$$
\left\{\begin{aligned}
-\omega^{3}-(b c+a c) \omega & =0 \\
-(a-c) \omega^{2}-2 a b c & =0 .
\end{aligned}\right.
$$

Later, equation (25) may derive a bifurcation surface as follows:

$$
\left\{\begin{array}{c}
a c^{2}+a b c+b c^{2}-a^{2} c=0 \\
\omega=\sqrt{(-b c-a c)},-b c-a c>0
\end{array}\right.
$$

Hence, from (14) and (26), we have obtained our three eigenvalues which gives as follows:

$$
\left\{\begin{array}{l}
\lambda_{1}=-(a-c) \\
\lambda_{2}=\omega i \\
\lambda_{3}=-\omega i .
\end{array}\right.
$$

Next, by finding the implicit derivative of $\lambda$ with respects to $b$ in equation (21), we have

$$
\lambda^{\prime}(b)=\frac{-c \lambda-2 a c}{3 \lambda^{2}+2(a-c) \lambda-(b c+a c)} .
$$

So, we investigated (28) to determine if Hopf bifurcation occurs by evaluating the following:

$$
\operatorname{Re} \lambda^{\prime}\left(b_{0}\right)=\frac{c(a+c)^{2}}{2\left(-3 a^{2} c-c^{2} a+c^{3}+a^{3}\right)}
$$


and

$$
\operatorname{Im} \lambda\left(b_{0}\right)=\omega=\sqrt{(-b c-a c)} \neq 0 .
$$

Theorem 3. When $b=b_{0}$ and $c<0$, there is an occurrence of Hopf bifurcation at the equilibrium points of $P_{2}=(\sqrt{(-b c)}, \sqrt{(-b c)}, b)$ and $P_{3}=$ $(-\sqrt{(-b c)},-\sqrt{(-b c)}, b)$ in the system (1).

Based on Theorem 3, we analysed the Hopf bifurcation of the Zhou system. So, we substituted the parameter value of $b$ with the critical value $b_{0}$. When $b=b_{0}$, the nontrivial equilibrium point has the coordinates

$$
x_{0}=\sqrt{\left(-\frac{a^{2}-a c}{a+c}\right) c}, y_{0}=\sqrt{\left(-\frac{a^{2}-a c}{a+c}\right) c}, z_{0}=\frac{a^{2}-a c}{a+c} .
$$

Then, we performed change of variables to translate the origin of the coordinates to the equilibrium point by

$$
\left\{\begin{array}{l}
x=X+x_{0} \\
y=Y+y_{0} \\
z=Z+z_{0}
\end{array}\right.
$$

From (32), the system (1) is transformed into

$$
\left\{\begin{array}{c}
\dot{X}=a(Y-X) \\
\dot{Y}=-\left(X Z-Z \sqrt{\left(-\frac{a^{2}-a c}{a+c}\right) c}\right) \\
\dot{Z}=X Y+(X+Y) \sqrt{\left(-\frac{a^{2}-a c}{a+c}\right) c}+c Z
\end{array}\right.
$$

We take a special case where we consider $c=-1$. Hence, we obtain the Jacobian matrix from the equation (33) and are transformed into the following:

$$
J=\left[\begin{array}{ccc}
-a & a & 0 \\
0 & 0 & -\sqrt{\frac{a^{2}+a}{a-1}} \\
\sqrt{\frac{a^{2}+a}{a-1}} & \sqrt{\frac{a^{2}+a}{a-1}} & -1
\end{array}\right] .
$$

Then, by using MAPLE 15 and some tedious calculations, we obtained the eigenvectors that we need

$$
m=\left(\begin{array}{c}
\frac{1}{\left.{ }^{\sqrt{ }} \overline{2} \sqrt{\frac{\mathrm{a}^{2}}{\mathrm{a}-1}}\right)} \\
\frac{a \sqrt{ } \overline{ } \overline{2} \sqrt{\frac{\mathrm{a}^{2}}{\mathrm{a}-1}}}{\sqrt{\frac{\mathrm{a}(\mathrm{a}+1)}{\mathrm{a}-1}}(a-1)}
\end{array}\right), m=\left(\begin{array}{c}
1 \\
\frac{\left(a-i^{\sqrt{ }} \overline{2} \sqrt{\frac{\mathrm{a}^{2}}{\mathrm{a}-1}}\right)}{a_{\sqrt{ }} \overline{2} \sqrt{\frac{\mathrm{a}^{2}}{\mathrm{a}-1}}} \\
\frac{2 a+(a-1) i}{\sqrt{\frac{\mathrm{a}(\mathrm{a}+1)}{\mathrm{a}-1}}(a-1)}
\end{array}\right),
$$




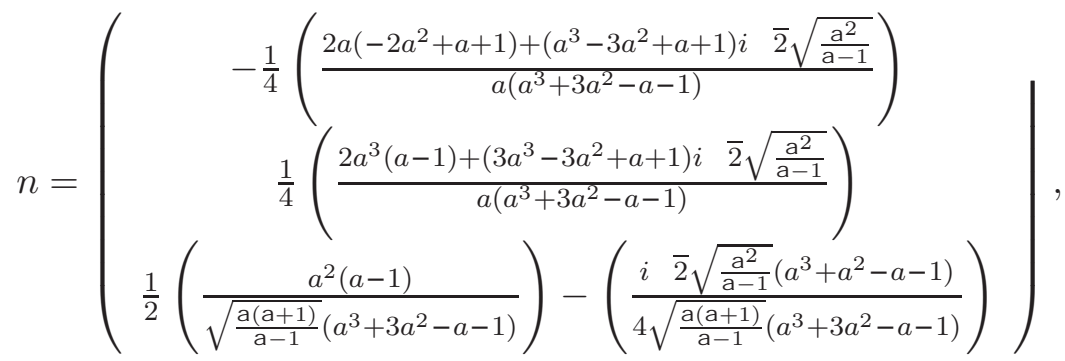

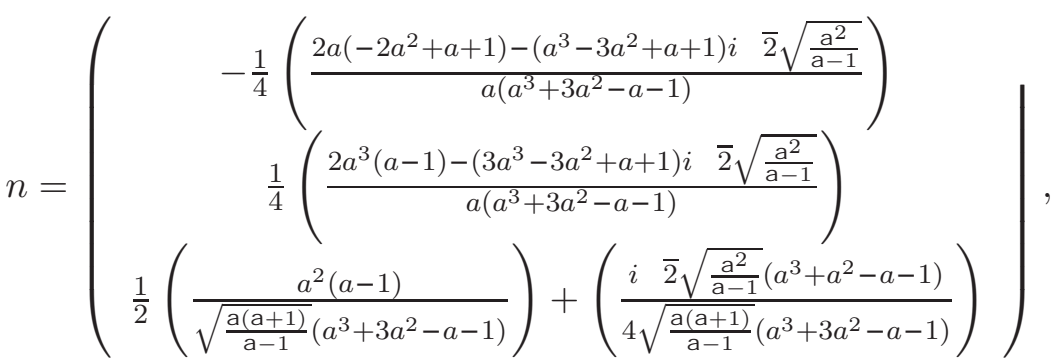

that satisfy

$$
J m=i \omega m, J m=-i \omega m, J^{T} n=-i \omega n, J^{T} n=i \omega n,\langle m, n\rangle=1 .
$$

Next, we will discuss the bilinear and trilinear terms in the dynamical system (1). It is obvious that in a dynamical system (1), there is only bilinear term. We define the bilinear term $B(\alpha, \beta)$ where $\alpha=\left(\alpha_{1}, \alpha_{2}, \alpha_{3}\right) \in \mathbb{R}^{2}$ and $\beta=\left(\beta_{1}, \beta_{2}, \beta_{3}\right) \in \mathbb{R}^{3}$. From (33), let

$$
\begin{aligned}
& F_{1}(X, Y, Z)=a(Y-X) \\
& F_{2}(X, Y, Z)=-\left(X Z-Z \sqrt{\left(-\frac{a^{2}-a c}{a+c}\right) c}\right) \\
& F_{3}(X, Y, Z)=X Y+(X+Y) \sqrt{\left(-\frac{a^{2}-a c}{a+c}\right) c}+c Z .
\end{aligned}
$$

Thus, the bilinear term can be expressed as:

$$
B(\alpha, \beta)=\left(0,-\alpha_{1} \beta_{3}-\alpha_{3} \beta_{1}, \alpha_{1} \beta_{2}+\alpha_{2} \beta_{1}\right)^{T} .
$$

Since there is no trilinear term in the dynamical system (1), the trilinear term $C(\alpha, \beta, \gamma)$ is equal to zero, i.e., it can be expressed as

$$
C(\alpha, \beta, \gamma)=(0,0,0)^{T}
$$

where $\alpha=\left(\alpha_{1}, \alpha_{2}, \alpha_{3}\right) \in \mathbb{R}^{2}, \beta=\left(\beta_{1}, \beta_{2}, \beta_{3}\right) \in \mathbb{R}^{3}$ and $\gamma=\left(\gamma_{1}, \gamma_{2}, \gamma_{3}\right) \in \mathbb{R}^{3}$. 
Next, we computed the value of $J^{-1}, B(m, m)$ and $B(m, m)$, yields

$$
\begin{aligned}
& J^{-1}=\left[\begin{array}{ccc}
\frac{-1}{2 a} & -\frac{(a-1)}{2 a(a+1)} & \frac{\sqrt{\frac{a(a+1)}{a-1}(a-1)}}{2 a(a+1)} \\
\frac{1}{2 a} & -\frac{(a-1)}{2 a(a+1)} & \frac{\sqrt{\frac{a(a+1)}{a-1}(a-1)}}{2 a(a+1)} \\
0 & -\frac{\sqrt{\frac{a(a+1)}{a-1}(a-1)}}{2 a(a+1)} & 0
\end{array}\right], \\
& B(m, m)=\left(\begin{array}{c}
0 \\
\frac{-2\left(2 a-(a-1) i \sqrt{2} \sqrt{\frac{\mathrm{a}^{2}}{\mathrm{a}-1}}\right)}{\sqrt{\frac{\mathrm{a}(\mathrm{a}+1)}{\mathrm{a}-1}(a-1)}} \\
\frac{2\left(a+i \sqrt{\sqrt{1}} \sqrt{\frac{\mathrm{a}^{2}}{\mathrm{a}-1}}\right)}{a}
\end{array}\right), \\
& B(m, m)=\left(\begin{array}{c}
0 \\
\frac{-4 a}{\sqrt{\frac{a(a+1)}{\mathrm{a}-1}(a-1)}} \\
2
\end{array}\right) .
\end{aligned}
$$

After that, we computed the following

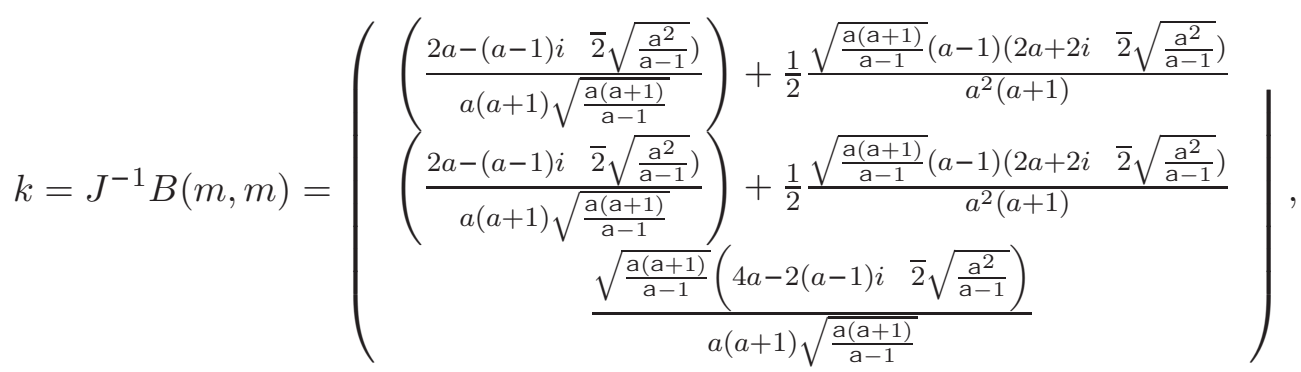

$$
\begin{aligned}
& B(m, k)=\left(\begin{array}{c}
0 \\
\frac{-2 a(3 a+7)+\left(3 a^{2}+2 a+9\right) i}{\sqrt{ }} \overline{2} \sqrt{\frac{\mathrm{a}^{2}}{\mathrm{a}-1}} \\
\frac{a(a+1)^{2}}{a\left(a^{2}+2 a-5\right)+(a+7)(a-1) i} \overline{2} \sqrt{\frac{\mathrm{a}^{2}}{\mathrm{a}-1}}
\end{array}\right), \\
& Q=\langle n, B(m, k)\rangle=\operatorname{Re}(Q)+i \operatorname{Im}(Q),
\end{aligned}
$$

where

$$
R e(Q)=\frac{\left(3 a^{6}-2 a^{5}+54 a^{4}-58 a^{3}+15 a^{2}+16 a\right)}{2 a^{2}\left(a^{3}+3 a^{2}-a-1\right)\left(a^{2}-1\right)}
$$




$$
\operatorname{Im}(Q)=\frac{\left(3 a^{6}+4 a^{5}+11 a^{4}-33 a^{3}+20 a^{2}-3 a-2\right) \sqrt{2} \sqrt{\frac{a^{2}}{a-1}}}{2 a^{2}\left(a^{3}+3 a^{2}-a-1\right)\left(a^{2}-1\right)} .
$$

Next, we calculated the inverse matrix of $(2 i \omega I-J)$ and obtained the following:

$$
(2 i w I-J)^{-1}=\left[\begin{array}{lll}
a_{11} & a_{12} & a_{13} \\
a_{21} & a_{22} & a_{23} \\
a_{31} & a_{32} & a_{33}
\end{array}\right]
$$

where

$$
\begin{gathered}
a_{11}=\frac{7 i a^{2}+2 \sqrt{2} \sqrt{\frac{a^{2}}{a-1}} a-i a-2 \sqrt{2} \sqrt{\frac{a^{2}}{a-1}}}{6\left(-i a+2 \sqrt{2} \sqrt{\frac{a^{2}}{a-1}}-i\right) a^{2}}, \\
a_{12}=-\frac{\left(2 \sqrt{2} \sqrt{\frac{a^{2}}{a-1}}-i\right)(a-1)}{6\left(-i a+2 \sqrt{2} \sqrt{\frac{a^{2}}{a-1}}-i\right) a}, \\
a_{13}=-\frac{i(a-1) \sqrt{\frac{a(a+1)}{a-1}}}{6\left(-i a+2 \sqrt{2} \sqrt{\frac{a^{2}}{a-1}}-i\right) a}, \\
a_{21}=-\frac{i(a+1)}{6\left(-i a+2 \sqrt{2} \sqrt{\frac{a^{2}}{a-1}}-i\right) a}, \\
a_{22}=-\frac{7 i a^{2}+2 \sqrt{2} \sqrt{\frac{a^{2}}{a-1}} a^{2}+i a-2 \sqrt{2} \sqrt{\frac{a^{2}}{a-1}}}{6\left(-i a+2 \sqrt{2} \sqrt{\frac{a^{2}}{a-1}}-i\right) a^{2}}, \\
a_{31}=-\frac{\left(2 \sqrt{2} \sqrt{\frac{a^{2}}{a-1}}-i a\right) \sqrt{\frac{a(a+1)}{a-1}}(a-1)}{6\left(-i a+2 \sqrt{2} \sqrt{\frac{a^{2}}{a-1}}-i\right) a^{2}}, \\
a_{23}=\frac{a\left(a+2 \sqrt{\frac{a(a+1)}{a-1}}(a-1)\right.}{\left.\sqrt{\frac{a^{2}}{a-1}}-i\right) a^{2}}
\end{gathered}
$$




$$
\begin{gathered}
a_{32}=\frac{\left(\sqrt{2} \sqrt{\frac{a^{2}}{a-1}}-i a\right) \sqrt{\frac{a(a+1)}{a-1}}(a-1)}{3\left(-i a+2 \sqrt{2} \sqrt{\frac{a^{2}}{a-1}}-i\right) a^{2}}, \\
a_{33}=\frac{\sqrt{2} \sqrt{\frac{a^{2}}{a-1}} a+4 i a-\sqrt{2} \sqrt{\frac{a^{2}}{a-1}}}{3\left(-i a+2 \sqrt{2} \sqrt{\frac{a^{2}}{a-1}}-i\right) a} .
\end{gathered}
$$

Then we found the following

$$
\begin{aligned}
& h=(2 i \omega I-J)^{-1} B(m, m)
\end{aligned}
$$

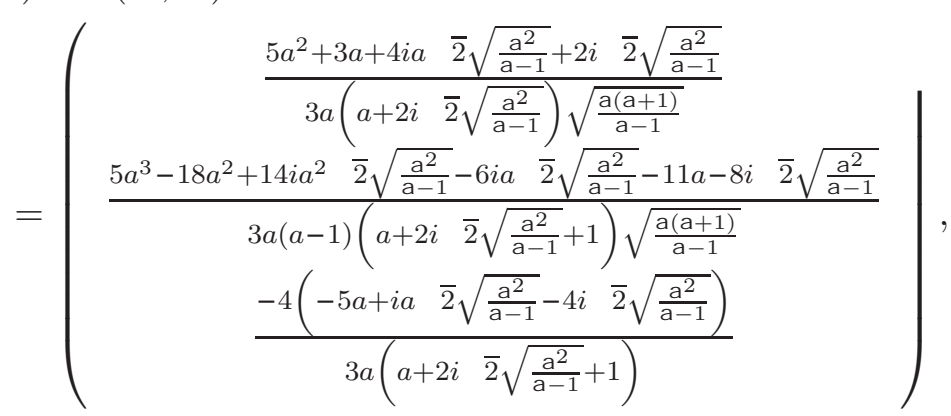

$$
\begin{aligned}
& B(m, h)=\left(\begin{array}{c}
0 \\
\frac{-(22 a+(a+17) i)^{\sqrt{ }} \overline{2} \sqrt{\frac{\mathrm{a}^{2}}{\mathrm{a}-1}}}{3 a\left(a+2 i^{\sqrt{ }} \overline{2} \sqrt{\frac{\mathrm{a}^{2}}{\mathrm{a}-1}}+1\right)} \\
\frac{2 a\left(5 a^{2}-6 a-5\right)+\left(13 a^{2}-6 a-7\right) i \sqrt{ } \overline{2} \sqrt{\frac{\mathrm{a}^{2}}{\mathrm{a}-1}}}{3 a(a-1)\left(a+2 i^{\sqrt{ }} \overline{2} \sqrt{\frac{\mathrm{a}^{2}}{\mathrm{a}-1}}+1\right) \sqrt{\frac{\mathrm{a}(\mathrm{a}+1)}{\mathrm{a}-1}}}
\end{array}\right) .
\end{aligned}
$$

Lastly, we obtained

$$
P=\langle n, B(m, h)\rangle=\operatorname{Re}(P)+i \operatorname{Im}(P)
$$

where

$$
\begin{gathered}
\operatorname{Re}(P)=-\frac{\left(13 a^{5}-55 a^{4}+35 a^{3}-6 a+5\right)}{3\left(a^{3}+9 a^{2}-a-1\right)\left(a^{3}+3 a^{2}-a-1\right)}, \\
\operatorname{Im}(P)=\frac{\sqrt{2} \sqrt{\frac{a^{2}}{a-1}}\left(3+3 a^{6}-11 a+29 a^{3}-20 a^{5}-29 a^{4}-11 a^{2}\right)}{3\left(a^{3}+9 a^{2}-a-1\right)\left(a^{3}+3 a^{2}-a-1\right)} .
\end{gathered}
$$


Therefore, we substituted $Q$ from (38) and $P$ from (42) into the first Lyapunov coefficient below:

$$
\begin{aligned}
l_{1}(0)= & \frac{1}{2 \omega} \operatorname{Re}\left[\langle n, C(m, m, m)\rangle-2\left\langle n, B\left(m, J^{-1} B(m, m)\right)\right\rangle\right. \\
& \left.+\left\langle n, B\left(m,(2 i \omega I-J)^{-1} B(m, m)\right)\right\rangle\right] \\
= & \frac{1}{2 \omega} \operatorname{Re}[-2 Q+P] \\
=- & \frac{\sqrt{2}\left(22 a^{8}+20 a^{7}+121 a^{6}+1336 a^{5}-1718 a^{4}+470 a^{3}+567 a^{2}-98 a-48\right)}{12 \sqrt{\frac{a^{2}}{a-1}} a\left(a^{3}+3 a^{2}-a-1\right)\left(a^{3}+9 a^{2}-a-1\right)\left(a^{2}-1\right)} .
\end{aligned}
$$

It is obvious that the equation (47) is always negative when the value of $a>1$. When $a<1$, the equation (47) has an imaginary value. When $a=1$, the equation (47) is undefined as the denominator will become zero. Hence, we have concluded in the following Theorem.

Theorem 4. The dynamical system (1) undergoes a supercritical Hopf bifurcation with the equilibrium points $P_{2}\left(x_{0}, y_{0}, z_{0}\right)$ and $P_{3}\left(-x_{0},-y_{0}, z_{0}\right)$ when $a>1$.

Based on Theorem 4, we have plotted the bifurcation phase of Zhou system (1.1) numerically. From (26), we substituted our value of $a=10$ and $c=-1$ to obtain the bifurcation value of $b$. So, we have obtained the values of $b$ from the following equations:

$$
\left\{\begin{array}{c}
a c^{2}+a b c+b c^{2}-a^{2} c=0 \\
9 b-110=0
\end{array}\right.
$$

Then, we solved equation (48) to get the values of $b=110 / 9$. The phase portraits of system (1) that undergoes Hopf bifurcation is plotted in Figure 3 for $b=110 / 9$.

\section{Conclusion}

In summary, we have studied the system (1) on Routh-Hurwitz Criterion to determine the behaviour of the system and the dynamical behavioural using Complementary-Cluster Energy-Barrier Criterion (CCEBC). Furthermore, we have used the method of [4] into proving that the system does have the occurrence of Hopf bifurcation. Finally, by first Lyapunov coefficient method [8], we 


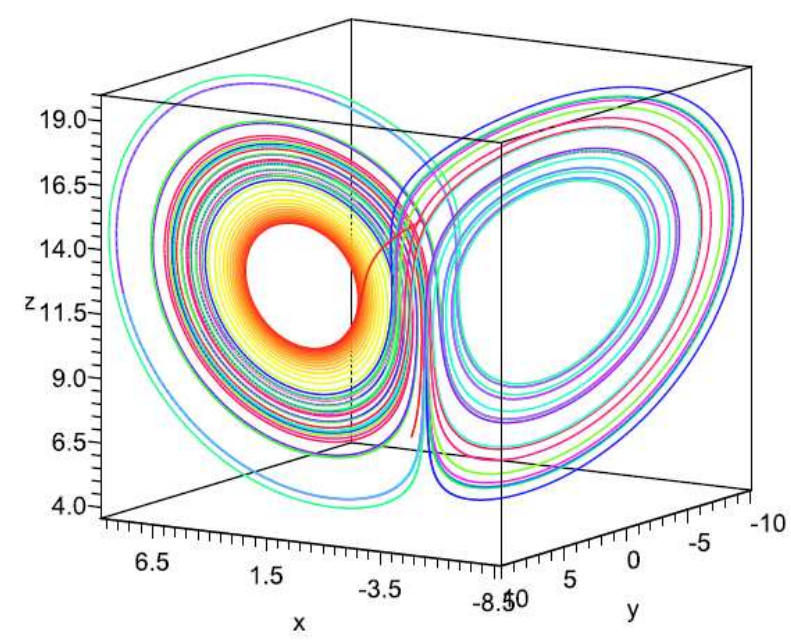

Figure 3: Phase portraits of system (1) when $a=10, c=-1$ and $b=110 / 9$.

have proved that the system has two points; point $P_{2}=(\sqrt{(-b c)}, \sqrt{(-b c)}, b)$ and $P_{3}=(-\sqrt{(-b c)},-\sqrt{(-b c)}, b)$, of which the Hopf bifurcations are supercritical when the value of $a$ greater than one.

\section{Acknowledgment}

The author gratefully acknowledges the Ministry of Education Malaysia, and Universiti Malaysia Terengganu that this research was partially supported under the Fundamental Research Grant Scheme (FRGS) 59347.

\section{References}

[1] Y.T. Ding, W.H. Jiang and H.B. Wang, Delayed feedback control and bifurcation analysis of Rossler chaotic system, Nonlinear Dynamics, 61 (2010), 707-715.

[2] Q.G. Gan, R. Xu and P.H. Yang, Bifurcation and chaos in a ratiodependent predator-prey system with time delay, Chaos, Solitons and Fractals, 39 (2009), 1883-1895. 
[3] F.R. Gantmacher, The Theory of Matrices, Chelsea Publishing Company, USA (1964).

[4] B.D. Hassard, N.D. Kazarinoff and Y.-H. Wan, Theory and Applications of Hopf Bifurcation, Cambridge University Press, London (1981).

[5] G.P. Hu and W.T. Li, Hopf bifurcation analysis for a delayed predator-prey system with diffusion effects, Nonlinear Analysis: Real World Applications, 11 (2010), 819-826.

[6] H.J. Hu and L.H. Huang, Stability and Hopf bifurcation in a delayed predator-prey system with stage structure for prey, Nonlinear Analysis: Real World Applications, 11 (2010), 2757-2769.

[7] E. Kreyszig, Introductory Functional Analysis with Applications, Wiley Classics Library, John Wiley \& Sons. Inc, USA (1978).

[8] Y.A. Kuznetsov, Elements of Applied Bifurcation Theory, Springer-Verlag, New York, Inc (1998).

[9] F. Li and Y.L. Jin, Hopf bifurcation analysis and numerical simulation in a 4D-hyperchaotic system, Nonlinear Dynamics, 67 (2012), 28572864. DOI 10.1007/s11071-011-0194-0.

[10] T.C. Li, G.R. Chen, Y. Tang and L.J. Yang, Hopf bifurcation of the generalized Lorenz canonical form, Nonlinear Dynamics, 47(4) (2007), 367-375.

[11] J.H. Lü, T.S. Zhou, G.R. Chen and S.C. Zhang, Local bifurcation of the Chen system, International Journal of Bifurcation and Chaos, 12(4) (2002), 2257-2270.

[12] U.A.M. Roslan, Z. Salleh and A. Kılıçman, Solving Zhou's chaotic system using Eulers's method, Thai Journal of Mathematics, 2 (2010), 299-309.

[13] U.A.M. Roslan, Z. Salleh and A. Kılıçman, Solving Zhou's chaotic system using fourth-order Runge-Kutta method, World Applied Sciences Journal, 21(6) (2013), 939-944.

[14] Y.L. Song and S.L. Yuan, Bifurcation analysis in a predator-prey system with time delay, Nonlinear Analysis, 7 (2006), 265-284.

[15] L.S. Tee and Z. Salleh, Dynamical analysis of a modified Lorenz system, Journal of Mathematics, vol. 2013, Article ID 820946, 8 pages, 2013. doi:10.1155/2013/820946. 
[16] L.S. Tee and Z. Salleh, Hopf bifurcation analysis of a modified Lorenz system, AIP Conference Proceedings, 1522 (2013), 158-167. doi: $10.1063 / 1.4801119$.

[17] X.J. Wu, J.S. Liu and G.R. Chen, Chaos synchonization of Rikitake chaotic attractor using the passive control technique, Nonlinear Dynamics, $\mathbf{5 3}$ (2008), 45-53.

[18] Z.Y. Yan, Hopf bifurcation in the Lorenz-type chaotic system, Chaos, Solitons and Fractals, 31 (2007), 1135-1142.

[19] C.H. Zhang, X.P. Yan and G.H. Cui, Hopf bifurcation in a predator-prey system with a discrete delay and a distributed delay, Nonlinear Analysis: Real World Applications, 11 (2010), 4141-4153.

[20] W.N. Zhou, Y.H. Xu, H.Q. Lu and L. Pan, On dynamics analysis of a new chaotic attractor, Physics Letters A, 372 (2008), 5773-5777. 